\title{
Leveraging Cone Double Description for Multi-contact Stability of Humanoids with Applications to Statics and Dynamics
}

\author{
Stéphane Caron* Quang-Cuong Pham ${ }^{\dagger}$ Yoshihiko Nakamura* \\ * Department of Mechano-Informatics, The University of Tokyo, Japan \\ $\dagger$ School of Mechanical and Aerospace Engineering, Nanyang Technological University, Singapore
}

\begin{abstract}
We build on previous works advocating the use of the Gravito-Inertial Wrench Cone (GIWC) as a general contact stability criterion (a "ZMP for non-coplanar contacts"). We show how to compute this wrench cone from the friction cones of contact forces by using an intermediate representation, the surface contact wrench cone, which is the minimal representation of contact stability for each surface contact. The observation that the GIWC needs to be computed only once per stance leads to particularly efficient algorithms, as we illustrate in two important problems for humanoids: "testing robust static equilibrium" and "time-optimal path parameterization". We show, through theoretical analysis and in physics simulations, that our method is more general and/or outperforms existing ones.
\end{abstract}

\section{INTRODUCTION}

Planning motions for legged robots requires taking into account three types of constraints : (i) avoid self-collisions and undesired collisions with the environment; (ii) respect actuator limits; (iii) keep balance (or avoid falling). While type (i) and type (ii) constraints are also experienced by fixed-base robots, type (iii) constraints are peculiar to legged robots and will constitute the main focus of this paper.

One way for legged robots to keep balance is to ensure that the links through which they make contact do not move with respect to the environment. A popular approach to guarantee this condition is to ensure that the Zero Moment Point (ZMP) be contained within the convex hull of the support area [1, 2]. This approach however presents several serious limitations:

- it only applies when all contacts are coplanar, and thus cannot be used when multiple non-coplanar contacts are involved;

- it only constraints 2 out of the 6 degrees of freedom (DoF) of a surface contact. More precisely, if the normal direction to the contact surface is denoted $\mathrm{Z}$, then the rotation around $\mathrm{X}$ and $\mathrm{Y}$ are constrained by the $\mathrm{ZMP}$ condition, but nothing can be said about translations along $\mathrm{X}, \mathrm{Y}, \mathrm{Z}$, as well as rotations around $\mathrm{Z}[3]$. This limitation is particularly serious at low friction [4, 5].

There have been many attempts to generalize the ZMP condition to overcome the above limitations. One approach is to consider individual contact forces distributed at the contact surfaces (see e.g., [6, 7, 8]), or surface contact wrenches [3], and require that these forces (or wrenches) satisfy the Coulomb condition. However, this approach lacks the concision and computational efficiency associated with the ZMP condition because of the large numbers of variables to consider (three times the number of individual contact forces or six times the number of contact wrenches).

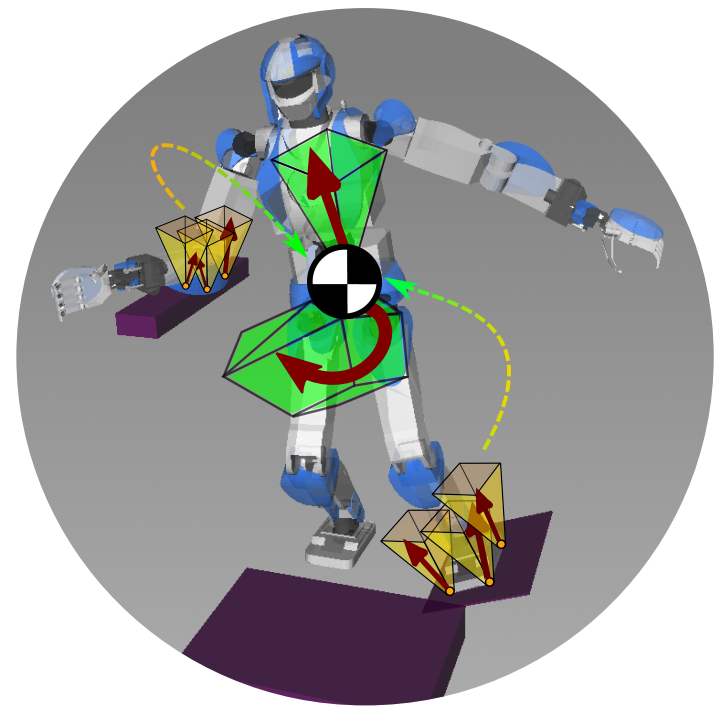

Fig. 1. One of the settings demonstrated in the paper: the humanoid climbs a box using a tilted support surface. Contact friction cones (for an arbitrary number of frictional contact points) are all combined into a single GravitoInertial Wrench Cone using the double description method. This representation is in turn converted to TOPP constraint vectors and the path retimed to get a highly dynamic, non-quasi-statically stable motion (i.e., which cannot be executed slowly). We checked the feasibility of the result in a physics simulator with simulated PID torque control.

Analyzing separately the unactuated part of the equation of motion (corresponding to the position and orientation of the base-link) reveals that, under the assumption that torque limits are sufficiently large, the stability of the contacts depends only on the gravito-inertial wrench [9]. This wrench, an object of dimension six, should lie in a convex cone (the Gravito-Inertial Wrench Cone or GIWC) generated by the contact constraints. This condition was proposed as a "universal stability criterion" by the authors of [6]. However, the representation of the gravito-inertial wrench that they used at that time was highly redundant (all contact forces as input variables) and their proof of stability was incorrect ${ }^{1}$. A derivation of the GIWC from contact forces was recently given in [10, 11], where the

\footnotetext{
${ }^{1}$ Equations (8) and (9) of [6] assume that the end-effector displacement $\Delta \boldsymbol{x}_{k}$ at contact $k$ is the same for all contacts, i.e., $\forall k, \Delta \boldsymbol{x}_{k}=\Delta \boldsymbol{x}_{G}$. This is true for single rigid bodies but false for articulated systems. For example, imagine a robot in contact with the floor, the ceiling, and two walls in front and behind it. The normal vectors are then $\boldsymbol{n}_{1}=(0,0,1), \boldsymbol{n}_{2}=$ $(0,0,-1), \boldsymbol{n}_{3}=(1,0,0)$ and $\boldsymbol{n}_{4}=(-1,0,0)$. Equation (8) would yield $\Delta \boldsymbol{x}_{G}=0$, i.e., the Center-of-Mass cannot move, but a redundantly-actuated humanoid can obviously move its CoM while making these four contacts.
} 
authors also proposed an algorithm to compute the GIWC using an efficient implementation of the double description method [12]. In both cases, the authors neglected the angular momentum: [10] assumed a point-mass model at the CoM, resulting in a simplified three-dimensional cone, while [11] dealt only with static equilibrium.

In the present paper, we propose to use an intermediate representation, the surface contact wrench [3], which eliminates the redundancy at the level of each surface contact and which constitutes a useful abstraction. Next, studying more closely the GIWC, we note that this cone only depends on the surface contact wrench cones and on the absolute positions and orientations of the contacts: in particular, it depends neither on the robot joint angles, nor on the position of its Centerof-Mass, nor on the direction of the gravity vector. Thus, the GIWC needs only be computed once per stance ${ }^{2}$. This observation enables us to propose more general and/or more efficient algorithms for the problems of "testing robust static equilibrium" and "time-optimal path parameterization".

\section{Relation to other works involving cone representations}

Using conversions between the face and span forms of a cone (hence the term "double description") to study contact stability was first suggested in [13]. In [14], other contact modes than the stable mode (for instance rolling, sliding, etc.) were studied by this method. However, the authors of these references were concerned with the problem of fixturing workpieces and did not consider the specificity of legged locomotion (e.g., surface contacts, gravito-inertial wrench, etc.) These works also relied on the cone algorithms implemented in [13], which is too slow to handle more than six contact points, and which have been superseded by recent advances in computational geometry [12]. In [3], the cone of surface contact wrenches was studied, but through algebraic manipulations rather than the double description method.

Another approach to deal with multi-dimensional cones consists in projecting them onto a plane using recursive polygon expansions [15, 7]. Note that this algorithm is peculiar to the dimension two and is not easily generalizable to higher dimensions ([15] does mention the possibility of generalization but we are not aware of any work in this direction). Nevertheless, it proved to be useful in the problems of "testing static equilibrium" [15] and "time-optimal path parameterization" [7, 8].

The above references highlight how crucial it is to find a suitable cone representation for a given problem. The 2Dpolygonal cross-section in [15] may be the minimum polyhedral representation when it comes to simple static equilibria, but it cannot account for robust static equilibria [16], which require computing $3 \mathrm{D}$ polyhedra.

In the problem of "time-optimal path parameterization" (TOPP), the works of [7, 8] project the set of possible

\footnotetext{
${ }^{2} \mathrm{~A}$ stance is the set of contact status for all possible contact links, along with the positions and orientations of the contacts. A change of stance occurs when e.g., a new link enters into contact with the environment, a pre-existing contact is broken, a sliding contact becomes a fixed contact, etc.
}

"motions" onto the $\left(\dot{s}^{2}, \ddot{s}\right)$ plane, where $s(t)$ denotes the position along a fixed path. The main limitation is that, contrary to [15], the projection needs to be performed at each discretized position $s$ along the path, and not simply once per stance. In the present communication, we show how, by using the GIWC and neglecting torque limits, one can decrease computation times from seconds to tens of milliseconds.

\section{Organization of the paper}

In Section III, we recall the relationship between stability of surface contacts and convex cones, as well as some basic definitions and results of polyhedral convex cone theory. In Section [III. we show how to compute the Gravito-Inertial Wrench Cone from the contact friction cones by using an intermediate representation, namely the surface contact wrench cone. We then use the combined GIWC to revisit two important problems arising in legged robotics: "testing robust static equilibrium" in Section IV, and "time-optimal path parameterization" in Section $\mathrm{V}]$ We validate the latter by a dynamic motion with non-coplanar contacts that we successfully run in a dynamics simulator. Finally, we discuss in Section VI some directions for further development.

\section{BACKGROUND}

\section{A. Stability of surface contacts}

As humanoid robots usually contact the environment through surfaces (e.g., the soles of their feet) rather than through points, we first recall some definitions and results regarding the stability of surface contacts (see [3] for more details).

Definition 1: In this paper, we consider the Coulomb friction model. A contact force $f$ is said valid when

- $f^{n} \geq 0$

- $\left\|\boldsymbol{f}^{t}\right\| \leq \mu f^{n}$

where $\mu$ is the friction coefficient, $f^{n}$ and $f^{t}$ denote respectively the normal and tangential components of the force with respect to a reference frame attached to the contact surface. In the following development, we will use the polyhedral approximation of the latter inequality, i.e.,

$$
\left|f_{x}\right| \leq \mu f^{n}, \quad\left|f_{y}\right| \leq \mu f^{n},
$$

where $\boldsymbol{f}^{t}=\left(f_{x}, f_{y}\right) . \triangle$

Physically speaking, a surface contact is a continuum of infinitesimal contact forces encoded by two fields: a scalar pressure field $p(x, y)$ and a two-dimensional stress field $\boldsymbol{\sigma}(x, y)$. Here, $(x, y)$ are 2D coordinates on the surface $\mathcal{S}$. The fields $p$ and $\sigma$ are the continuous analogues of $f^{n}$ and $f^{t}$.

Definition 2: The pair of fields $(p(x, y), \boldsymbol{\sigma}(x, y))$ is said valid when, for any $(x, y) \in \mathcal{S}$,

- $p(x, y) \geq 0$

- $\|\boldsymbol{\sigma}(x, y)\| \leq \mu p(x, y) \triangle$

When the environment and contacting link are rigid, the interaction between them is fully described by a contact 
wrench $\boldsymbol{w}=(\boldsymbol{f}, \boldsymbol{\tau})$ where

$$
\begin{aligned}
& \boldsymbol{f} \stackrel{\text { def }}{=} \int_{\mathcal{S}} \boldsymbol{\nu}(x, y) \mathrm{d} x \mathrm{~d} y, \\
& \boldsymbol{\tau} \stackrel{\text { def }}{=} \int_{\mathcal{S}} \overrightarrow{O C_{x y}} \wedge \boldsymbol{\nu}(x, y) \mathrm{d} x \mathrm{~d} y,
\end{aligned}
$$

with the three-dimensional vectors $\nu \stackrel{\text { def }}{=}(\sigma, p)$ and $\overrightarrow{O C_{x y}} \stackrel{\text { def }}{=}$ $(x, y, 0)$. We say that the field $\boldsymbol{\nu}$ sums up to $\boldsymbol{w}$, which get us to the following definition

Definition 3: A contact wrench $\boldsymbol{w}$ is said valid if there exist valid fields that sum up to $\boldsymbol{w}$. $\triangle$

Assume that the convex hull of the contact area is a convex polygon. Then the following proposition shows that, as far as the validity of the wrench is concerned, it is sufficient to consider forces at the vertices of the polygon instead of the continuous fields.

Proposition 1: A contact wrench $\boldsymbol{w}$ is valid if and only if ${ }^{3}$ there exist valid contact forces at the vertices of the polygon that sum up to $\boldsymbol{w}$. $\triangle$

Consider now a robot with $n$ actuated joints making $m$ surface contacts with the environment. The equation of motion of the robot is given by

$$
\mathbf{M}(\boldsymbol{q}) \ddot{\boldsymbol{q}}+\boldsymbol{h}(\boldsymbol{q}, \dot{\boldsymbol{q}})=\mathbf{S}^{\top} \boldsymbol{\tau}_{a}+\sum_{\text {contact } i} \mathbf{J}_{i}^{\top} \boldsymbol{w}_{i}
$$

where $\boldsymbol{q}$ is a vector of dimension $n+6$ describing the configuration of the robot (the first $n$ coordinates for the actuated joints, the last 6 coordinates for the position and orientation of the base link), $\mathbf{S}$ is a $n \times(n+6)$ selection matrix, $\mathbf{J}_{i}$ is the $6 \times(n+6)$ contact Jacobian for contact $i$ (i.e., the Jacobian for the transformation of the surface reference frame), $\boldsymbol{w}_{i}$ is the contact wrench for contact $i$ (expressed in the surface frame).

The relationship between valid contact wrenches and (weak [17, 14]) contact stability is illustrated by the following

Definition 4 (Feasibility under stable contacts): Assume that the robot is in a given state $(\boldsymbol{q}, \dot{\boldsymbol{q}})$ where $\mathbf{J}_{i} \dot{\boldsymbol{q}}=0$ for all contacts $i$. Then an acceleration $\ddot{\boldsymbol{q}}$ is feasible under stable contacts if (1) $\mathbf{J}_{i} \ddot{\boldsymbol{q}}=-\mathbf{H}_{i} \dot{\boldsymbol{q}}$ and (2) there exist torques $\boldsymbol{\tau}_{a}$ within the torque limits and valid contact wrenches $\boldsymbol{w}_{i}$ that satisfy the equation of motion (3) $\triangle$

In other words, wrench and torque validity are the dynamic inequalities coupled with the kinematic binding of the positions of contacting links. (See [17, 14, 3] for more discussion on contact stability.) As mentioned in the Introduction, we shall always make the assumption that torque limits are sufficiently large, so that our focus is on the existence of valid contact wrenches. In Section III] we shall characterize this existence by a single stability criterion: the Gravito-Inertial Wrench Cone.

\section{B. Cone double description}

We recall here some definitions and results in the theory of Polyhedral Convex Cones. For more details, the reader is

\footnotetext{
${ }^{3}$ The "if" part is true when one authorizes Dirac fields. If one does not authorize Dirac fields, whether this implication is true is still an open question.
}

referred to [13, 14]. From now on, all the "cones" we mention will implicitly be polyhedral.

Definition 5: A polyhedral convex cone $\mathcal{C}$ is defined by a set of inequalities

$$
\mathcal{C}=\operatorname{face}\left(\boldsymbol{u}_{1}, \ldots, \boldsymbol{u}_{m}\right)=\left\{\boldsymbol{x}: \boldsymbol{u}_{1}^{\top} \boldsymbol{x} \leq 0, \ldots, \boldsymbol{u}_{m}^{\top} \boldsymbol{x} \leq 0\right\} .
$$

for some vectors $\left(\boldsymbol{u}_{1}, \ldots, \boldsymbol{u}_{m}\right) . \triangle$

Cones can be equivalently defined as positive combination of a family of base vectors

Theorem 1 (Weyl-Minkowski): For every polyhedral convex cone $\mathcal{C}$, there exists a set of vectors $\left(\boldsymbol{v}_{1}, \ldots, \boldsymbol{v}_{n}\right)$ such that

$\mathcal{C}=\operatorname{span}\left(\boldsymbol{v}_{1}, \ldots, \boldsymbol{v}_{n}\right)=\left\{\sum_{i=1}^{n} z_{i} \boldsymbol{v}_{i}: z_{1} \geq 0 \ldots z_{n} \geq 0\right\} . \triangle$

If one stacks the $\boldsymbol{u}_{i}^{\top}$ horizontally into a matrix $\mathbf{U}$ and the $\boldsymbol{v}_{i}$ vertically into a matrix $\mathbf{V}$, the above definitions become in matrix form (vector inequalities are element-wise)

$$
\begin{aligned}
\mathcal{C} & =\operatorname{face}(\mathbf{U})=\{\boldsymbol{x}: \mathbf{U} \boldsymbol{x} \leq \mathbf{0}\} \\
& =\operatorname{span}(\mathbf{V})=\{\mathbf{V} \boldsymbol{z}: \boldsymbol{z} \geq \mathbf{0}\}
\end{aligned}
$$

The span and face forms have each their own advantages. It is trivial to test whether a vector $\boldsymbol{x}$ belongs to a cone face $(\mathbf{U})$ (suffices to check $\mathbf{U} \boldsymbol{x} \leq \mathbf{0}$ ) but the same operation requires solving a linear program in span form. Meanwhile, the span form is compatible with linear combinations : if $\boldsymbol{y}=\mathbf{A} \boldsymbol{x}$, then $\boldsymbol{x}$ belongs to the cone $\operatorname{span}(\mathbf{V})$ if and only if $\boldsymbol{y}$ belongs to the cone $\operatorname{span}(\mathbf{A V})$. Doing the same in face form is a more involved operation.

An important result in polyhedral cone theory is the possibility to convert between the face and span forms of a cone (hence the term "double description"), which we shall use extensively in Section III

Proposition 2: Given a matrix U, one can compute explicitly a matrix $\mathbf{U}^{S}$ such that $\operatorname{span}\left(\mathbf{U}^{S}\right)=$ face $(\mathbf{U})$.

Given a matrix $\mathbf{V}$, one can compute explicitly a matrix $\mathbf{V}^{F}$ $\operatorname{such}$ that face $\left(\mathbf{V}^{F}\right)=\operatorname{span}(\mathbf{V}) \triangle$

An algorithm to compute $\mathbf{U}^{S}$ and $\mathbf{V}^{F}$ was developed in [13]. Its complexity is exponential - the problem being NPcomplete - and in practice, computation times are prohibitive when the number of span or face vectors is greater than 20 . In our implementation, we used the codd library [12], which performs fast conversions even for more than 100 span or face vectors (see e.g., Tables $\mathrm{I}$ and $\mathrm{II}$ ).

\section{Computing the Gravito-Inertial Wrench Cone USING DOUBLE DESCRIPTION}

We now show how to compute the Gravito-Inertial Wrench Cone using surface contact wrenches. For each link $k$, denote by $\boldsymbol{p}_{k}$ the position of its Center-of-Mass (CoM) in the laboratory frame, $\mathbf{R}_{k}$ the orientation matrix of the link frame with respect to the laboratory frame, $\boldsymbol{\omega}_{k}$ the angular velocity of the link in the link frame, $\mathbb{I}_{k}$ the inertia matrix of the link in the link frame. Let $\boldsymbol{p}_{\mathrm{CoM}}$ be the position of the CoM of the robot, and $\mathcal{L}$, its angular momentum calculated with respect 


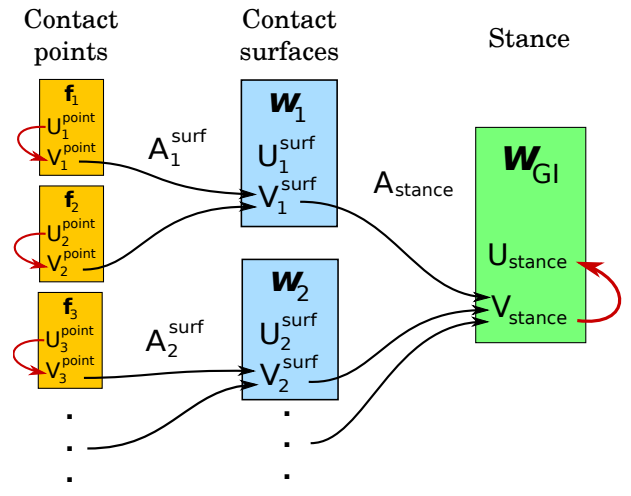

Fig. 2. Outline of our approach to compute the face form of the GIWC Matrices $\mathbf{U}$ and $\mathbf{V}$ are associated with respectively the face and span forms of the cones. Black arrows denote matrix multiplication while red arrows denote uses of the double description algorithm.

to $\boldsymbol{p}_{\mathrm{CoM}}$, i.e.,

$$
\begin{aligned}
\boldsymbol{p}_{\mathrm{CoM}} & \stackrel{\text { def }}{=} \frac{1}{m} \sum_{\text {link } k} m_{k} \boldsymbol{p}_{k}, \\
\mathcal{L} & \stackrel{\text { def }}{=} \sum_{\operatorname{link} k}\left(\boldsymbol{p}_{k}-\boldsymbol{p}_{\mathrm{CoM}}\right) \times m_{k} \dot{\boldsymbol{p}}_{k}+\mathbf{R}_{k} \mathbb{I}_{k} \boldsymbol{\omega}_{k} .
\end{aligned}
$$

Definition 6: The gravito-inertial wrench $\boldsymbol{w}_{\mathrm{GI}} \stackrel{\text { def }}{=}$ $\left(\boldsymbol{f}_{\mathrm{GI}}, \boldsymbol{\tau}_{\mathrm{GI}}\right)$, computed with respect to the origin of the laboratory frame, is defined by

$$
\begin{array}{lll}
\boldsymbol{f}_{\mathrm{GI}} & \stackrel{\text { def }}{=} & m\left(\boldsymbol{g}-\ddot{\boldsymbol{p}}_{\mathrm{CoM}}\right), \\
\boldsymbol{\tau}_{\mathrm{GI}} \stackrel{\text { def }}{=} & \boldsymbol{p}_{\mathrm{CoM}} \times m\left(\boldsymbol{g}-\ddot{\boldsymbol{p}}_{\mathrm{CoM}}\right)-\dot{\mathcal{L}} .
\end{array}
$$

Definition 7 (and Proposition): The set of gravito-inertial wrenches that correspond to feasible joint accelerations under given stable contacts (Definition 4) is a cone, called the Gravito-Inertial Wrench Cone (GIWC).

Our approach can be summarized as follows (see also Fig. 2). First, we convert the face representation of the friction cones for individual contact forces into their span form. In span form, combining the individual cones into the surface wrench cones is immediate. We thus get one contact wrench cone for every contacting link.

Next, based on the equation of motion for the free-floating coordinates, we express the linear mapping between all contact wrenches and the gravito-inertial wrench. Applying this mapping to the contact wrench cones gives us the GIWC in span form. Finally, we convert the span form of the GIWC to its face form, which can be readily used in e.g., QP or TOPP solvers. Note that only the last two steps need to be executed at each change of stance.

\section{A. Span form of the surface contact wrench cone}

We first show the following proposition.

Proposition 3: Assuming polyhedral Coulomb friction, the set of valid contact wrenches is a polyhedral convex cone. Its expression depends only on the surface geometry and friction model. $\triangle$
Proof: Consider a valid wrench $\boldsymbol{w}$. By Proposition 1 , there exists a set of valid contact forces $\boldsymbol{f}_{j}$ at the vertices of the polygon that sum up to $\boldsymbol{w}$. The summation procedure is linear and can thus be encoded by a matrix $\mathbf{A}_{\text {surf }}$, i.e., $\boldsymbol{w}=\mathbf{A}_{\text {surf }} \boldsymbol{f}_{\text {all }}$, where $f_{\text {all }}$ is the stacked vector of all the contact forces $\boldsymbol{f}_{j}$. On the other hand, the validity of the contact forces of Definition 1 can be put in the following matrix form (assuming polyhedral approximation) $\mathbf{U}_{\text {point }} \boldsymbol{f}_{\text {all }} \leq \mathbf{0}$. It follows that $\boldsymbol{w} \in \operatorname{span}\left(\mathbf{A}_{\text {surf }} \mathbf{U}_{\text {point }}^{S}\right)$.

Conversely, consider a $\boldsymbol{w} \in \operatorname{span}\left(\mathbf{A}_{\text {surf }} \mathbf{U}_{\text {point }}^{S}\right)$, which means that there exists $\boldsymbol{z} \geq 0$ such that $\boldsymbol{w}=\mathbf{A}_{\text {surf }} \mathbf{U}_{\text {point }}^{S} \boldsymbol{z}$. Let $\boldsymbol{f}=\mathbf{U}_{\text {point }}^{S} \boldsymbol{z}$. Since $\boldsymbol{z} \geq 0, \boldsymbol{f} \in \operatorname{span}\left(\mathbf{U}_{\text {point }}^{S}\right)=$ face $\left(\mathbf{U}_{\text {point }}\right)$. Thus $\mathbf{U}_{\text {point }} \boldsymbol{f} \leq 0$. Combined with the fact that $\boldsymbol{w}=\mathbf{A}_{\text {surf }}\left(\mathbf{U}_{\text {point }}^{S} \boldsymbol{z}\right)=\mathbf{A}_{\text {surf }} \boldsymbol{f}$, this shows that $\boldsymbol{w}$ is valid.

One can also note $\mathbf{V}_{\text {point }} \stackrel{\text { def }}{=} \mathbf{U}_{\text {point }}^{S}$ and $\mathbf{V}_{\text {surf }} \stackrel{\text { def }}{=}$ $\mathbf{A}_{\text {surf }} \mathbf{U}_{\text {point }}^{S}=\mathbf{A}_{\text {surf }} \mathbf{V}_{\text {point }}$. The surface wrench cone is the minimal representation of the contact constraint, in the sense that its dimension (six) is equal to the contact DoF [3]. Note that $\mathbf{V}_{\text {surf }}$ can be reduced at this stage by eliminating the columns that can be written as non-negative combinations of the others. Note also that, as the matrix $\mathbf{V}_{\text {surf }}$ depends only on the surface geometry and friction coefficient, its computation and reduction need to be done only once for all.

\section{B. From surface contact wrenches to gravito-inertial wrench}

The last six equations of the robot dynamics (3) can be formulated as [9]

$$
\begin{aligned}
m \ddot{\boldsymbol{p}}_{\mathrm{CoM}} & =\sum_{\text {contact } i} \mathbf{R}_{i} \boldsymbol{f}_{i}+m \boldsymbol{g}, \\
\dot{\mathcal{L}} & =\sum_{\text {contact } i}\left(\boldsymbol{p}_{i}-\boldsymbol{p}_{\mathrm{CoM}}\right) \times \mathbf{R}_{i} \boldsymbol{f}_{i}+\mathbf{R}_{i} \boldsymbol{\tau}_{i} .
\end{aligned}
$$

One can next rewrite equations (8) and (9) in terms of the gravito-inertial wrench

$$
\begin{aligned}
\boldsymbol{f}_{\mathrm{GI}} & =-\sum_{\text {contact } i} \mathbf{R}_{i} \boldsymbol{f}_{i}, \\
\boldsymbol{\tau}_{\mathrm{GI}} & =-\sum_{\text {contact } i} \boldsymbol{p}_{i} \times \mathbf{R}_{i} \boldsymbol{f}_{i}+\mathbf{R}_{i} \boldsymbol{\tau}_{i} .
\end{aligned}
$$

In matrix form, this is equivalent to

$$
\boldsymbol{w}_{\mathrm{GI}}=\sum_{\text {contact } i}\left[\begin{array}{cc}
-\mathbf{R}_{i} & \mathbf{0} \\
-\widehat{\boldsymbol{p}}_{i} \mathbf{R}_{i} & -\mathbf{R}_{i}
\end{array}\right] \boldsymbol{w}_{i}=\mathbf{A}_{\text {stance }} \boldsymbol{w}_{\text {all }},
$$

where $\boldsymbol{w}_{\text {all }}$ is the stacked vector of the contact wrenches $\boldsymbol{w}_{i}$. The key observation here is that $\mathbf{A}_{\text {stance }}$ only depends on the $\boldsymbol{p}_{i}$ and $\mathbf{R}_{i}$, which are fixed for each stance.

Proposition 4: The gravito-inertial wrench is linearly determined by the contact wrenches. Furthermore, this linear mapping only depends on the absolute positions and orientations of the contacting links. $\triangle$

\section{Face form of the GIWC}

We can now prove the following proposition

Proposition 5: The set of gravito-inertial wrenches that correspond to feasible joint accelerations is a polyhedral convex 
cone, which depends only on (1) the absolute positions and orientations of the contacting links, and (2) the local geometry and friction properties at the contact surfaces. The face form of this cone can be explicitly computed. $\triangle$

Proof: From Proposition 3, the contact wrench $\boldsymbol{w}_{i}$ is valid if and only if $\boldsymbol{w}_{i} \in \operatorname{span}\left(\mathbf{V}_{i}^{\text {surf }}\right)$, which implies that $\boldsymbol{w}_{\text {all }} \in \operatorname{span}\left(\mathbf{V}_{\text {all }}\right)$ where

$$
\mathbf{V}_{\text {all }} \stackrel{\text { def }}{=}\left[\begin{array}{ccc}
\mathbf{V}_{1}^{\text {surf }} & \mathbf{0} & \mathbf{0} \\
\mathbf{0} & \ddots & \mathbf{0} \\
\mathbf{0} & \mathbf{0} & \mathbf{V}_{N}^{\text {surf }}
\end{array}\right] .
$$

Thus, $\boldsymbol{w}_{\mathrm{GI}}=\mathbf{A}_{\text {stance }} \boldsymbol{w}_{\text {all }} \in \operatorname{span}\left(\mathbf{A}_{\text {stance }} \mathbf{V}_{\text {all }}\right)$. Define now $\mathbf{U}_{\text {stance }} \stackrel{\text { def }}{=}\left(\mathbf{A}_{\text {stance }} \mathbf{V}_{\text {all }}\right)^{F}$. Then, there exist valid wrenches at the contacts if and only if $\mathbf{U}_{\text {stance }} \boldsymbol{w}_{\mathrm{GI}} \leq \mathbf{0}$.

Since $\mathbf{A}_{\text {stance }}$ depends only on the $\boldsymbol{p}_{i}$ and $\mathbf{R}_{i}, \mathbf{U}_{\text {stance }}$ depends only on (1) the absolute positions $\boldsymbol{p}_{i}$ and orientations $\mathbf{R}_{i}$ of the contacting links, and (2) the local geometry and friction properties at the contact surfaces. Thus, it needs to be computed only once per stance.

\section{TEST OF ROBUST STATIC EQUILIBRIUM}

A robot is in static equilibrium if, at zero velocity and acceleration, the gravity wrench can be "generated" by valid contact wrenches. If the terrain is flat, static equilibrium is achieved if the CoM of the robot lies above the convex hull of the robot's feet (support area), or in other words, if the CoM belongs to an infinite right cylinder whose axis is parallel to gravity and whose cross-section is the support area. If the terrain is not flat, then the CoM positions that induce static equilibrium are still an infinite right cylinder with axis parallel to gravity, but whose cross-section is no longer the support area. The computation of the cross-section is given in [15]. Once this cross-section is computed, one can test static equilibrium quickly by checking whether the $(x, y)$ coordinates of the CoM is within the cross-section.

Static equilibrium is said to be robust if not only the gravity wrench, but any wrench in some neighborhood around the gravity wrench can be generated by valid contact wrenches [16]. In some limit situations, while simple static equilibrium may be satisfied (e.g., when the CoM is at the boundaries of the support polygon in flat terrain), robust static equilibrium adds an informed safety margin. Uncertainties such as model inaccuracies or unknown disturbances can be modeled within this framework.

In [16], the authors studied robust equilibrium for a robot in a 2D environment (one horizontal and one vertical directions) and for a polytopic neighborhood. They showed that the set of $\mathrm{CoM}$ positions that induce robust static equilibrium is no longer an infinite vertical band, but a polygon. They also gave a "line sweep" algorithm to compute that polygon. Here, we show that the face representation of the GIWC given in Section III-C provides a fast test for robust static equilibrium in $3 D$, which generalizes both [15] (which discussed only simple equilibrium) and [16] (which discussed only the 2D case).
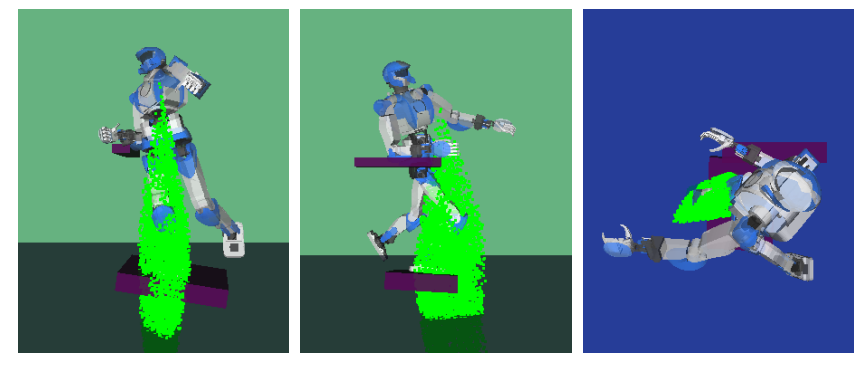

Fig. 3. Testing robust static equilibrium. The robot right arm is supported by the ledge while its right foot is supported by an inclined box. The desired neighborhood around the gravity wrench is associated with $\left\{\boldsymbol{g}_{1}, \boldsymbol{g}_{2}, \boldsymbol{g}_{3}, \boldsymbol{g}_{4}\right\}=$ $\{(0.15,0, g),(-0.15,0, g),(0,0.15, g),(0,-0.15, g)\}$. We sampled one million random $\mathrm{CoM}$ positions, those of which satisfy robust static equilibrium are depicted by a green dot. The aggregate of green dots outlines the shape of the robust static equilibrium wrench cone. The three images show views from the $\mathrm{X}, \mathrm{Y}$ and $\mathrm{Z}$ directions respectively.

In the case of static equilibrium, we have $\ddot{p}_{\mathrm{CoM}}=\mathbf{0}$ and $\dot{\mathcal{L}}=\mathbf{0}$. Equations 8 and 9 become

$$
\begin{aligned}
& \mathbf{0}=\sum_{\text {contact } i} \mathbf{R}_{i} \boldsymbol{f}_{i}+m \boldsymbol{g}, \\
& \mathbf{0}=\sum_{\text {contact } i}\left(\boldsymbol{p}_{i}-\boldsymbol{p}_{\mathrm{CoM}}\right) \times \mathbf{R}_{i} \boldsymbol{f}_{i}+\mathbf{R}_{i} \boldsymbol{\tau}_{i},
\end{aligned}
$$

i.e. $\left(m \boldsymbol{g}, m \boldsymbol{p}_{\mathrm{CoM}} \times \boldsymbol{g}\right)=\mathbf{A}_{\text {stance }} \boldsymbol{w}_{\text {all }}$.

We are now interested not only in the generation of the gravity wrench $\left(m \boldsymbol{g}, m \boldsymbol{p}_{\mathrm{CoM}} \times \boldsymbol{g}\right)$ but also in that of any wrench in a polytopic neighborhood $\mathcal{N}\left(\boldsymbol{p}_{\mathrm{CoM}}\right)$ around it. Consider for simplicity the neighborhood defined as the convex hull of $\left\{\left(m \boldsymbol{g}_{1}, m \boldsymbol{p}_{\mathrm{CoM}} \times \boldsymbol{g}_{1}\right), \ldots,\left(m \boldsymbol{g}_{K}, m \boldsymbol{p}_{\mathrm{CoM}} \times \boldsymbol{g}_{K}\right)\right\}$ where $\boldsymbol{g}_{1}, \ldots, \boldsymbol{g}_{K}$ are vectors around $\boldsymbol{g}$.

Consider the sets $\mathcal{M}_{k}$ defined as

$$
\mathcal{M}_{k} \stackrel{\text { def }}{=}\left\{\boldsymbol{p}: \mathbf{U}_{\text {stance }}\left(\begin{array}{c}
\boldsymbol{g}_{k} \\
\boldsymbol{p} \times \boldsymbol{g}_{k}
\end{array}\right) \geq \mathbf{0}\right\}
$$

and their intersection $\mathcal{M} \stackrel{\text { def }}{=} \bigcap_{k \in[1, K]} \mathcal{M}_{k}$. We show that $\mathcal{M}$ is the set of CoM positions that ensure robust static equilibrium.

Proof: assume that $\boldsymbol{p} \in \mathcal{M}$. Consider a wrench $\boldsymbol{w}^{*} \in \mathcal{N}(\boldsymbol{p})$. There exists $\lambda_{1} \geq 0, \ldots, \lambda_{k} \geq 0$ such that

$$
\boldsymbol{w}^{*}=m\left(\lambda \boldsymbol{g}_{1}+\ldots \lambda \boldsymbol{g}_{K}, \boldsymbol{p} \times\left(\lambda \boldsymbol{g}_{1}+\ldots \lambda \boldsymbol{g}_{K}\right)\right) .
$$

Since $\boldsymbol{p} \in \mathcal{M}$, we have that, for all $k$,

$$
\mathbf{U}_{\text {stance }}\left(\begin{array}{c}
\boldsymbol{g}_{k} \\
\boldsymbol{p} \times \boldsymbol{g}_{k}
\end{array}\right) \geq \mathbf{0}
$$

thus there exist valid contact wrenches $\boldsymbol{w}_{\mathrm{all}_{1}}, \ldots, \boldsymbol{w}_{\mathrm{all}_{K}}$ such that $\left(-m \boldsymbol{g}_{k},-m \boldsymbol{p} \times \boldsymbol{g}_{k}\right)=\mathbf{A}_{\text {stance }} \boldsymbol{w}_{\mathrm{all}_{k}}$. By convexity, the contact wrench $\boldsymbol{w}_{\text {all }}=\sum_{k} \lambda_{k} \boldsymbol{w}_{\text {all }_{k}}$ is valid. On the other hand, by linearity, we have $\boldsymbol{w}^{*}=\mathbf{A}_{\text {stance }} \boldsymbol{w}_{\text {all }}$. Together, these last two equations show that the wrench $\boldsymbol{w}^{*}$ can be generated. Since $\boldsymbol{w}^{*}$ is arbitrary in $\mathcal{N}(\boldsymbol{p})$, this implies that $\boldsymbol{p}$ induces robust equilibrium.

Conversely, assume that $\boldsymbol{p}$ induces robust equilibrium, i.e., all wrenches $\boldsymbol{w} \in \mathcal{N}(\boldsymbol{p})$ can be generated. In particu- 
lar there exists a valid contact wrench $\boldsymbol{w}_{\text {all }_{1}}$ that generates $\left(m \boldsymbol{g}_{1}, m \boldsymbol{p} \times \boldsymbol{g}_{1}\right)$. By construction of $\mathbf{U}_{\text {stance }}$, this implies that $p \in \mathcal{M}_{1}$. By repeating this reasoning, one can show that $\boldsymbol{p} \in \mathcal{M}_{2}, \ldots, \boldsymbol{p} \in \mathcal{M}_{K}$, which implies that $\boldsymbol{p} \in \mathcal{M}$.

It can be noted that the $\mathcal{M}_{k}$ are infinite right prisms with axis parallel to $\boldsymbol{g}_{k}$. Thus, if the $\boldsymbol{g}_{k}$ are not collinear, then $\mathcal{M}$ will not be a right prism, but a polyhedron. Nevertheless, from the development presented above, testing robust static equilibrium requires simply to pre-compute once $\mathbf{U}_{\text {stance, }}$ and subsequently, for each candidate CoM position, to evaluate $K$ matrix multiplications and comparisons as given in (12) Note that the algorithm in [15] would require to perform $K$ polytope projections in the pre-computation phase.

Fig. 3 illustrates the proposed robust equilibrium test. For two surface contacts (right arm and right foot), the matrix $\mathbf{U}_{\text {stance }}$ had dimension $105 \times 6$ and could be computed in $3.5 \mathrm{~ms}$ on our 8 -core $3.00 \mathrm{GHz}$ microprocessor. Subsequently, each test took $0.1 \mathrm{~ms}$.

\section{Time-Optimal Path Parameterization (TOPP)}

Consider a path $\mathcal{P}$ - represented as the underlying path of a trajectory $\boldsymbol{p}(s)_{s \in\left[0, s_{\text {end }}\right]}$ - in the configuration space. Assume that $\boldsymbol{p}(s)_{s \in\left[0, s_{\text {end }}\right]}$ is $C^{1}$ - and piecewise $C^{2}$-continuous. We are interested in time-parameterizations of $\mathcal{P}$, which are increasing scalar functions $s:[0, T] \rightarrow\left[0, s_{\text {end }}\right]$, under kinodynamic constraints. If the constraints can be expressed in the form

$$
\ddot{s} \boldsymbol{a}(s)+\dot{s}^{2} \boldsymbol{b}(s)+\boldsymbol{c}(s) \leq 0,
$$

then there exists efficient methods and implementations to find the time-optimal parameterization $s(t)$ (see [18] and references therein).

\section{A. TOPP reduction}

Reducing constraints to the form 13 is relatively straightforward for fully-actuated systems, but harder for overactuated systems, which include closed-chain manipulators or legged robots with more than one surface contact (see e.g., [19]). In [7], the author adapts the 2D projection algorithm of [15] to achieve this reduction. However, the projection needs to be performed at each discretized position $s$ along the path, which is time-consuming. Using the development of [III. we now present a method that needs cone computations only once per stance; for each discretized position $s$, only some matrix multiplications are required to compute the vectors $\boldsymbol{a}(s), \boldsymbol{b}(s), \boldsymbol{c}(s)$.

Consider a path $\boldsymbol{p}(s)$ of the CoM (note that we have dropped the subscript CoM for simplicity). Differentiating twice, we have

$$
\ddot{\boldsymbol{p}}=\boldsymbol{p}_{s} \ddot{s}+\boldsymbol{p}_{s s} \dot{s}^{2} .
$$

The angular momentum $\mathcal{L}$ can be expressed in Jacobian form $\mathcal{L}=\mathbf{J}_{\mathcal{L}}(\boldsymbol{q}) \dot{\boldsymbol{q}}=\mathbf{J}_{\mathcal{L}}(s) \boldsymbol{q}_{s} \dot{s}$. Therefore, one can always write

$$
\dot{\mathcal{L}}=l_{1} \ddot{s}+l_{2} \dot{s}^{2},
$$

for some functions $l_{1}$ and $l_{2}$. In general, there is no function $l$ such that $l_{1}=l_{s}$ and $l_{2}=l_{s s}$ (in other words, $\mathcal{L}$ is not integrable). Exceptions include the cases when $\mathcal{L}=0$ or when $\mathcal{L}$ is the angular momentum of a single rigid body.

Substituting the expressions of $\ddot{p}$ and $\dot{\mathcal{L}}$ into (6) and (7), we have

$$
\boldsymbol{w}_{\mathrm{GI}}=\left(\begin{array}{c}
m\left(\boldsymbol{g}-\boldsymbol{p}_{s} \ddot{s}-\boldsymbol{p}_{s s} \dot{s}^{2}\right) \\
m \boldsymbol{p} \times\left(\boldsymbol{g}-\boldsymbol{p}_{s} \ddot{s}-\boldsymbol{p}_{s s} \dot{s}^{2}\right)-\boldsymbol{l}_{1} \ddot{s}-\boldsymbol{l}_{2} \dot{s}^{2}
\end{array}\right) .
$$

Thus, the condition $\mathbf{U}_{\text {stance }} \boldsymbol{w}_{\mathrm{GI}} \leq \mathbf{0}$ can be rewritten as

$$
\begin{array}{r}
-\ddot{s} \mathbf{U}_{\text {stance }}\left(\begin{array}{c}
m \boldsymbol{p}_{s} \\
m \boldsymbol{p} \times \boldsymbol{p}_{s}+\boldsymbol{l}_{1}
\end{array}\right)-\dot{s}^{2} \mathbf{U}_{\text {stance }}\left(\begin{array}{c}
m \boldsymbol{p}_{s s} \\
m \boldsymbol{p} \times \boldsymbol{p}_{s s}+\boldsymbol{l}_{2}
\end{array}\right) \\
+\mathbf{U}_{\text {stance }}\left(\begin{array}{c}
m \boldsymbol{g} \\
m \boldsymbol{p} \times \boldsymbol{g}
\end{array}\right) \leq \mathbf{0},
\end{array}
$$

which is in the canonical form of 13

\section{B. Trajectory generation}

As equation (14) illustrates, the centroidal trajectory, i.e., the joint trajectory of the linear and angular momenta $(m \dot{\boldsymbol{p}}(t), \mathcal{L}(t)))$, is the only piece of information required to formulate the contact stability constraint. Our first attempts were therefore to interpolate a centroidal trajectory, re-time it with TOPP to satisfy contact constraints, and then interpolate a whole-body trajectory with the same linear and angular momenta by Inverse Kinematics (IK). This approach is however hampered by the difficulty in interpolating the angular momentum $\mathcal{L}(t)$. Because of its non-holonomy, it is impossible to integrate it into a position variable, as is the case with the linear momentum. We experimented with the suggestion from [20] to regulate $\mathcal{L}=0$, but it resulted in large free limb movements with a tendency to get the limbs in inconvenient positions (e.g., hands behind the back), making the overall control task harder.

We subsequently opted for a different pipeline: interpolate the CoM and end-effector trajectories first, compute a corresponding whole-body trajectory $\boldsymbol{q}(s)$ by inverse kinematics, and finally enforce contact stability along this trajectory by TOPP. The angular momentum $\mathcal{L}\left(\boldsymbol{q}, \boldsymbol{q}_{s}\right)$ will then result from the configurations computed by the IK solver.

Numerical TOPP solvers require relatively smooth velocity and acceleration profiles. Discontinuities in velocity or acceleration are allowed and properly dealt with [18], but we found that the acceleration profiles returned by a velocity-based IK solver are too erratic for proper use with TOPP. To avoid this, we used an acceleration-based IK solver. The wholebody trajectory $\boldsymbol{q}(s)$ is computed as the double-integral of an acceleration trajectory $\ddot{\boldsymbol{q}}(s)$, where accelerations are computed as solutions to the following QP problem. Provided a reference trajectory $\boldsymbol{p}(s)$ and $\boldsymbol{f}_{\text {link }}(s)$ for the CoM and steered noncontacting link, minimize

$$
\begin{aligned}
& w_{\mathrm{CoM}}\left\|\mathbf{J}_{\mathrm{CoM}} \boldsymbol{q}_{s s}+\gamma\left(\dot{\boldsymbol{p}}^{*}(t)-\mathbf{J}_{\mathrm{CoM}} \boldsymbol{q}_{s}\right)+\boldsymbol{q}_{s}^{\top} \mathbf{H}_{\mathrm{CoM}} \boldsymbol{q}_{s}\right\|^{2} \\
& +w_{\text {link }}\left\|\mathbf{J}_{\text {link }} \boldsymbol{q}_{s s}+\gamma\left(f_{\text {link }}^{*}(t)-\mathbf{J}_{\text {link }} \boldsymbol{q}_{s}\right)+\boldsymbol{q}_{s} \mathbf{H}_{\text {link }} \boldsymbol{q}_{s}\right\|^{2},
\end{aligned}
$$

such that

(1) $\forall c \in$ contacts, $\mathbf{J}_{c} \dot{\boldsymbol{q}}_{s s}=-\gamma \mathbf{J}_{c} \dot{\boldsymbol{q}}_{s}+\dot{\boldsymbol{q}}_{s}^{\top} \mathbf{H}_{c} \dot{\boldsymbol{q}}_{s}$, and

(2) $K_{s s}\left(\dot{\boldsymbol{q}}_{\min }-\dot{\boldsymbol{q}}_{s}\right) \leq \dot{\boldsymbol{q}}_{s s} \leq K_{s s}\left(\dot{\boldsymbol{q}}_{\max }-\dot{\boldsymbol{q}}_{s}\right)$, 


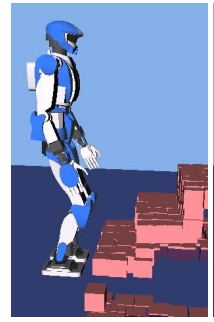

$t=0.0 \mathrm{~s}$

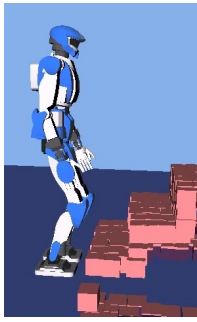

$t=0.4 \mathrm{~s}$

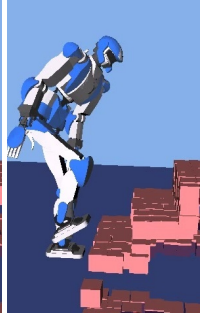

$t=0.8 \mathrm{~s}$

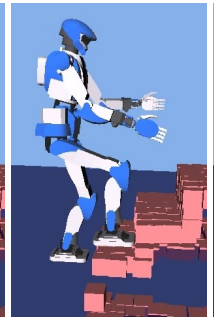

$t=1.2 \mathrm{~s}$

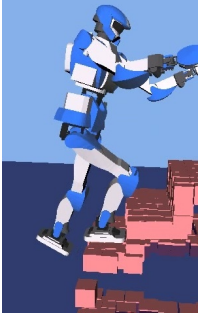

$t=1.6 \mathrm{~s}$

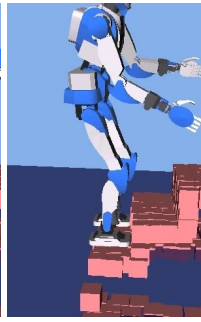

$t=2.0 \mathrm{~s}$

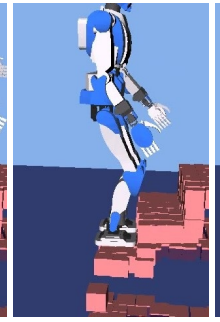

$t=2.4 \mathrm{~s}$

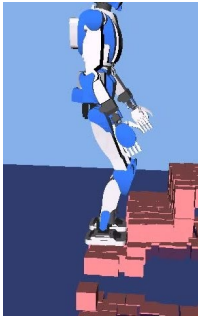

$t=2.8 \mathrm{~s}$

Fig. 4. Stair climbing motion where contact stability is checked using our method. Time stamps are shown under each frame. The retimed motion is very fast as all underlying configurations are all statically stable.

where $\dot{\boldsymbol{q}}_{\text {max }} \stackrel{\text { def }}{=}-K_{s}\left(\boldsymbol{q}-\boldsymbol{q}_{\max }\right)$ and $\dot{\boldsymbol{q}}_{\min } \stackrel{\text { def }}{=}-K_{s}\left(\boldsymbol{q}-\boldsymbol{q}_{\min }\right)$. Here, $\mathbf{J}_{\star}\left(\right.$ resp. $\left.\mathbf{H}_{\star}\right)$ denote the Jacobians (resp. Hessians) of constraints, where constraint labels are $c$ for contact, CoM for the center-of-mass and link for the free end-effector. This problem can be readily addressed by many off-the-shelf QP solvers. We used CVXOPT ${ }^{4}$ which is free software and could deal efficiently with both equality and inequality constraints. The reader is referred to [21] for a more general solution to QP-based inverse kinematics.

In all experiments, we used $K_{s}=K_{s s}=\gamma=10 \mathrm{~s}^{-1}$, $w_{\text {CoM }}=1$ and $w_{\text {link }}=0.1$. CoM and end-effector trajectories were interpolated as simple line segments.

\section{Experiment 1: stair climbing}

We first illustrate our method on a common stair climbing motion. The staircase (red boxes, reconstructed from point cloud data) has a step height of $24 \mathrm{~cm}$. The motion is quasistatically stable (it can be executed at arbitrary slow velocities) and alternates single and double support segments where the projection of the CoM is moved linearly from one support foot to the other. The retimed motion is shown in Figure 4. Computation times are reported in Table II, where we detail the three consecutive computations : that of the gravito-inertial wrench matrix $\mathbf{U}_{\text {stance }}\left(T_{\mathbf{U}_{\text {stance }}}\right)$, of the constraint vectors $\boldsymbol{a}(s), \boldsymbol{b}(s), \boldsymbol{c}(s)\left(T_{\boldsymbol{a b c}}\right)$ and of the re-timing by our numerical TOPP solver ( $\left.T_{\text {TOPP }}\right)$.

TABLE I

COMPUTATION TIMES (MS) FOR THE STAIR CLIMBING MOTION, AVERAGED OVER 10 RUNS ON AN 8-CORE 3.00 GHZ PROCESSOR. SEgMENTS ARE IDENTIFIED by their time STAMPS (SEe Figure 4 ).

\begin{tabular}{|c|r|c|c|c|}
\hline Segment $(\mathrm{s})$ & Size of $\mathbf{U}_{\text {stance }}$ & $T_{\mathbf{U}_{\text {stance }}}$ & $T_{\boldsymbol{a b c}}$ & $T_{\text {TOPP }}$ \\
\hline $0.0-0.4$ & $114 \times 6$ & 4.2 & 2.0 & 130 \\
$0.4-1.0$ & $16 \times 6$ & 1.3 & 2.0 & 350 \\
$1.0-1.2$ & $162 \times 6$ & 4.5 & 2.2 & 560 \\
$1.2-2.0$ & $16 \times 6$ & 1.3 & 2.0 & 360 \\
$2.0-2.8$ & $162 \times 6$ & 4.3 & 2.2 & 150 \\
\hline Total & - & 15.6 & 10.4 & 1,550 \\
\hline
\end{tabular}

\section{Experiment 2: box climbing using tilted support}

We now apply our method to the setting depicted in Figure 1. The robot climbs a $10-\mathrm{cm}$ box using two contacts : its right arm, set on a 90-cm high horizontal ledge, and its left foot, set

\footnotetext{
${ }^{4}$ http://cvxopt.org/
}

on a $25^{\circ}$ inclined stepping surface. The motion generated in this experiment may seem unnatural, as the robot could step on the box directly. Yet, the motivation for this setting is twofold. First, stability throughout this motion cannot be checked by ZMP, as frictional contacts may be lost (the "sufficient friction" assumption does not apply) and the contact surfaces non-coplanar. Second, the solution to this constrained problem is not feasible at low velocities, which means CoM-based methods such as [15] cannot be applied.

Figure 5 shows the execution of the motion in our physics simulator. In this scenario, the friction coefficient of environment bodies (respectively the floor, box, arm support and inclined plane) was set to $\mu=0.9$. Due to the high speed of the retimed segment (the combined duration of the right-foot and left-foot steps is only two seconds), the position controller was not responsive enough to track the reference trajectory exactly. To palliate this, we made time-optimal trajectories slower by using a smaller friction coefficient $\mu=0.7$ in the computations of the GIWC for TOPP (namely, $\mu=0.7$ and contact surfaces scale by 0.7 ) in order to get a "safer" motion.

A further difficulty, compared to other experiments were humanoids walk on slightly inclined surfaces, was that we could not use the stabilizer module of the humanoid, which is designed for horizontal floors. Thus, the motion represented in Figure 5 was run with pure open-loop position control.

The transition between the first and second foot steps is the most challenging part of the motion. Its configurations are not statically stable, and it therefore needs a non-zero minimum velocity to be performed without falling. As CoM trajectories are straight lines in our design, the problem of finding a feasible whole-body motion for this segment boiled down to finding a suitable CoM velocity $\boldsymbol{v}$. Manual trials being unsuccessful, we chose this velocity heuristically as $\boldsymbol{v}^{*}=\arg \max _{\boldsymbol{v}} \beta_{\boldsymbol{v}}(0,1)$, where $\beta$ is an internal vector field from TOPP representing the maximum acceleration achievable along the path (see [18] for details). This heuristic provided feasible solutions in practice. Furthermore, from sampling neighboring vectors, we estimated the solid angle of valid CoM velocity vectors around $\boldsymbol{v}^{*}$ to 0.1 steradian, i.e., less than $0.8 \%$ of the orientation space.

\section{E. Comparison to previous work}

Tables $\mathrm{II}$ and $\mathrm{I}$ show the performances of our method on the box and stair climbing motions. We compare these perfor- 


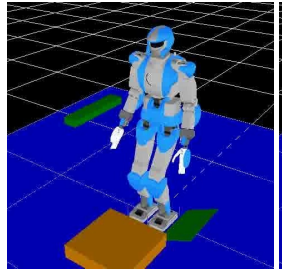

$t=3.9 \mathrm{~s}$

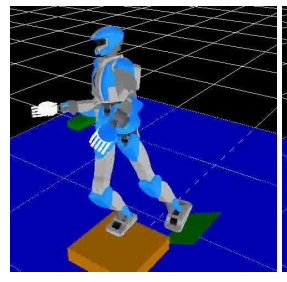

$t=15.6 \mathrm{~s}$

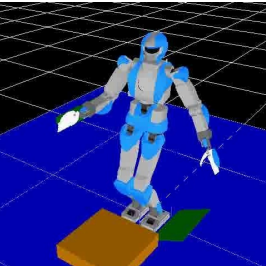

$t=6.7 \mathrm{~s}$

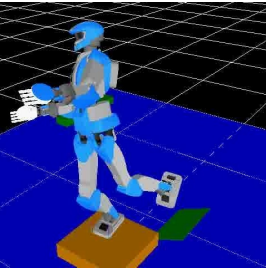

$t=15.8 \mathrm{~s}$

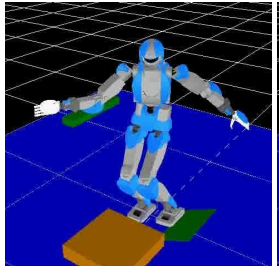

$t=10.6 \mathrm{~s}$

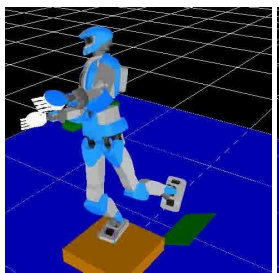

$t=16.1 \mathrm{~s}$

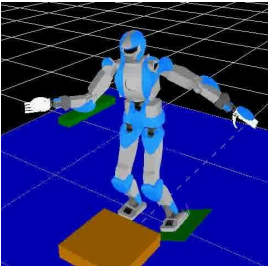

$t=13.3 \mathrm{~s}$

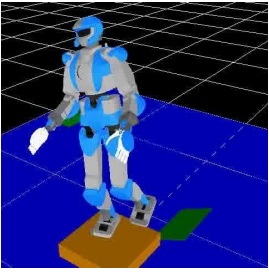

$t=19.7 \mathrm{~s}$

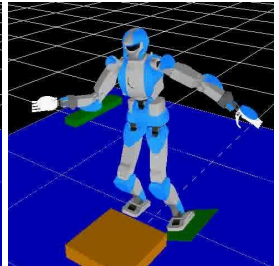

$t=15.0 \mathrm{~s}$

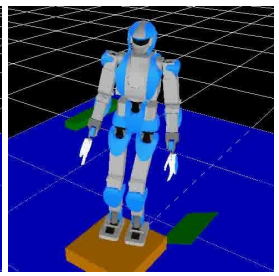

$t=21.1 \mathrm{~s}$

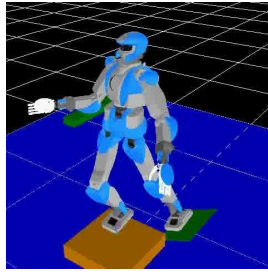

$t=15.3 \mathrm{~s}$

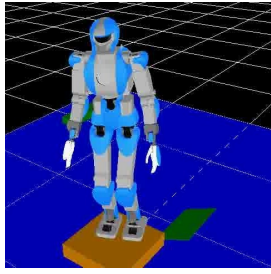

$t=21.4 \mathrm{~s}$

Fig. 5. Execution of the multi-contact whole-body trajectory on a humanoid robot in a physics simulator. Time stamps are written under each frame. The dynamic part of the motion occurs between $15 \mathrm{~s}$ and $16 \mathrm{~s}$, where movements need to be fast in order to maintain contact stability. This segment is dynamically but not quasi-statically stable: at least one contact is lost when the motion is executed at lower velocities.

TABLE II

COMPUTATION TIMES (MS) FOR THE BOX CLIMBING MOTION, AVERAGED OVER 10 RUNS ON AN 8-CORE $3.00 \mathrm{GHZ}$ PROCESSOR. SEGMENTS ARE IDENTIFIED BY THEIR TIME STAMPS (SEE FIGURE 5 ).

\begin{tabular}{|c|r|c|c|c|}
\hline Segment $(\mathrm{s})$ & Size of $\mathbf{U}_{\text {stance }}$ & $T_{\mathbf{U}_{\text {stance }}}$ & $T_{\boldsymbol{a b c}}$ & $T_{\text {TOPP }}$ \\
\hline $0.0-6.7$ & $113 \times 6$ & 4.4 & 2.4 & 420 \\
$6.7-15.0$ & $84 \times 6$ & 3.7 & 2.5 & 310 \\
$15.0-15.3$ & $55 \times 6$ & 3.0 & 2.5 & 60 \\
$15.3-21.4$ & $118 \times 6$ & 4.0 & 2.5 & 320 \\
\hline Total & - & 15.1 & 9.9 & 1,110 \\
\hline
\end{tabular}

mances with those reported in previous work by Hauser [7] where the recursive polygon expansion algorithm is used to retime humanoid trajectories under distributed contact forces and actuator limits. The two main differences between this work and our method are that (1) we calculate the GIWC rather than a recursive polygon expansion, and (2) we use a numerical TOPP solver rather than a Sequential Linear Programming (SLP) solver. In order to make run times more comparable, we used the same path discretization resolution $N=100$

In accordance with previous work, we break down computation times as follows

- Pre-computation of feasible sets $\left(T_{\mathbf{U}_{\text {stance }}}+T_{\boldsymbol{a b c}}\right)$ : the time reported in [7] is $2.40 \mathrm{~s}$, while our solution takes around $30 \mathrm{~ms}$ in both settings to perform this operation. When actuator torque is not limiting, it is therefore one to two orders of magnitude faster than previous work.

- Computation of the velocity profile (TTOPP): the time reported in [7] is $2.46 \mathrm{~s}$, while our solution takes between $1 \mathrm{~s}$ and $1.5 \mathrm{~s}$ in the previous climbing motions. Two factors are concurring here to put execution times on the same magnitude: we use a numerical TOPP solver, an approach that is usually orders of magnitude faster than SLP; however, we do not prune redundant inequalities, which is a side benefit of recursive polygon expansion.
Overall, our approach cuts down to tens of milliseconds the pre-computations where previous work would spent half of the computing budget, while having comparable performances on the rest of the time-optimal re-timing. This comparison should of course be taken with care, as our robot, task, and computing environment are different from [7]. What we point out here is the difference in orders of magnitude on the computation times of feasible sets alone.

\section{CONCLUSION}

In this paper, we derived the Gravito-Inertial Wrench Cone from surface-wrench contact constraints. Maintaining the gravito-inertial wrench within this cone is known as a general stability criterion, a "generalized ZMP". Armed with this condition, we revisited two important problems arising in humanoid robotics. In the problem of "testing robust static equilibrium", we extended the results of [15, [16] to threedimensional static equilibrium. In the problem of "timeoptimal path parameterization" (TOPP), we showed a new reduction of the contact constraints to the classical form which requires cone computations only once per stance, thereby dramatically improving the performance of TOPP in multicontact [7, 8]. We compared our computation times to the state-of-the-art, and validated our results by realistic simulated motions on a full-size humanoid model.

There are many directions for further development. Our derivation for time-optimal control can be directly integrated into the framework of Admissible Velocity Propagation [22] to perform non-quasi-static motion planning under contact changes. Another prospect is that, although we focused on stable contacts in this work, the surface contact wrench cones from Section III-A can be used to express arbitrary contact modes: stable, sliding, rolling, etc.

Besides these extensions, we believe that the wrench cone condition developed through the works of [13, 14, 9, 6, 10] and in the present paper has the potential to be to multi-contact stability what the ZMP is to planar locomotion. 


\section{REFERENCES}

[1] M. Vukobratovic, B. Borovac, and D. Surdilovic, "Zeromoment point-proper interpretation and new applications," in IEEE/RAS International Conference on $\mathrm{Hu}$ manoid Robots, 2001.

[2] A. Goswami, "Postural stability of biped robots and the foot-rotation indicator (fri) point," The International Journal of Robotics Research, vol. 18, no. 6, pp. 523533, 1999.

[3] S. Caron, Q.-C. Pham, and Y. Nakamura, "Stability of surface contacts for humanoid robots: Closed-form formulae of the contact wrench cone for rectangular support areas," in Robotics and Automation (ICRA), 2015 IEEE International Conference on, (To Appear in). IEEE, 2015.

[4] B. Ugurlu, J. A. Saglia, N. G. Tsagarakis, and D. G. Caldwell, "Yaw moment compensation for bipedal robots via intrinsic angular momentum constraint," International Journal of Humanoid Robotics, vol. 9, no. 04, 2012.

[5] R. Cisneros, K. Yokoi, and E. Yoshida, "Yaw moment compensation by using full body motion," in Mechatronics and Automation (ICMA), 2014 IEEE International Conference on. IEEE, 2014, pp. 119-125.

[6] H. Hirukawa, S. Hattori, K. Harada, S. Kajita, K. Kaneko, F. Kanehiro, K. Fujiwara, and M. Morisawa, "A universal stability criterion of the foot contact of legged robotsadios zmp," in Robotics and Automation, 2006. ICRA 2006. Proceedings 2006 IEEE International Conference on. IEEE, 2006, pp. 1976-1983.

[7] K. Hauser, "Fast interpolation and time-optimization with contact," The International Journal of Robotics Research, vol. 33, no. 9, pp. 1231-1250, 2014.

[8] Q.-C. Pham and O. Stasse, "Time-optimal path parameterization for redundantly-actuated robots: A numerical integration approach," IEEE/ASME Transactions on Mechatronics, 2015.

[9] P.-B. Wieber, "Holonomy and nonholonomy in the dynamics of articulated motion," in Fast motions in biomechanics and robotics. Springer, 2006, pp. 411-425.

[10] Z. Qiu, A. Escande, A. Micaelli, and T. Robert, "Human motions analysis and simulation based on a general criterion of stability," in First International Symposium on Digital Human Modeling, 2011.

[11] A. Escande, A. Kheddar, and S. Miossec, "Planning contact points for humanoid robots," Robotics and Autonomous Systems, vol. 61, no. 5, pp. 428-442, 2013.

[12] K. Fukuda and A. Prodon, "Double description method revisited," in Combinatorics and computer science. Springer, 1996, pp. 91-111.

[13] S. Hirai, "Analysis and planning of manipulation using the theory of polyhedral convex cones," Ph.D. dissertation, Kyoto University, 1991.

[14] D. J. Balkcom and J. C. Trinkle, "Computing wrench cones for planar rigid body contact tasks," The International Journal of Robotics Research, vol. 21, no. 12, pp.
1053-1066, 2002.

[15] T. Bretl and S. Lall, "Testing static equilibrium for legged robots," Robotics, IEEE Transactions on, vol. 24, no. 4, pp. 794-807, 2008.

[16] Y. Or and E. Rimon, "Computation and graphical characterization of robust multiple-contact postures in twodimensional gravitational environments," The International Journal of Robotics Research, vol. 25, no. 11, pp. 1071-1086, 2006.

[17] J.-S. Pang and J. Trinkle, "Stability characterizations of rigid body contact problems with coulomb friction," ZAMM-Journal of Applied Mathematics and Mechanics/Zeitschrift für Angewandte Mathematik und Mechanik, vol. 80, no. 10, pp. 643-663, 2000.

[18] Q.-C. Pham, "A general, fast, and robust implementation of the time-optimal path parameterization algorithm," IEEE Transactions on Robotics, vol. 30, pp. 1533-1540, 2014.

[19] L. Righetti, J. Buchli, M. Mistry, M. Kalakrishnan, and S. Schaal, "Optimal distribution of contact forces with inverse-dynamics control," The International Journal of Robotics Research, vol. 32, no. 3, pp. 280-298, 2013.

[20] M. Popovic, A. Hofmann, and H. Herr, "Angular momentum regulation during human walking: biomechanics and control," in Robotics and Automation, 2004. Proceedings. ICRA'04. 2004 IEEE International Conference on, vol. 3. IEEE, 2004, pp. 2405-2411.

[21] A. Escande, N. Mansard, and P.-B. Wieber, "Hierarchical quadratic programming: Fast online humanoid-robot motion generation," The International Journal of Robotics Research, pp. 1006-1028, 2014.

[22] Q.-C. Pham, S. Caron, and Y. Nakamura, "Kinodynamic planning in the configuration space via velocity interval propagation," in Robotics: Science and System, 2013. 